\title{
Monte Carlo Simulations of the R/1 Automated Damage Test
}

\author{
M. Runkel
}

This paper was prepared for submittal to the 30th Boulder Damage Symposium: Annual Symposium on Optical Materials for High Power Lasers Boulder, Colorado

September 28 - October 1, 1998

September 18, 1998

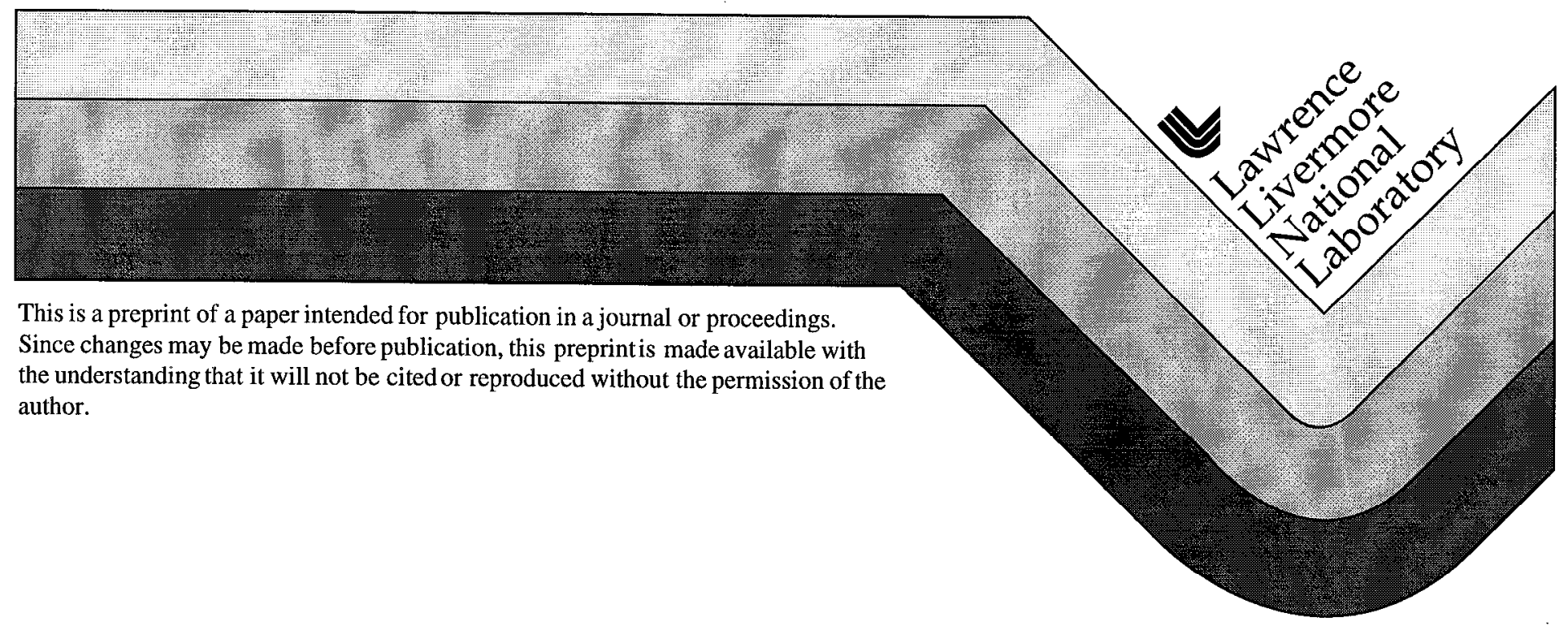




\section{DISCLATMER}

This document was prepared as an account of work sponsored by an agency of the United States Government. Neither the United States Government nor the University of California nor any of their employees, makes any warranty, express or implied, or assumes any legal liability or responsibility for the accuracy, completeness, or usefulness of any information, apparatus, product, or process disclosed, or represents that its use would not infringe privately owned rights. Reference herein to any specific commercial product, process, or service by trade name, trademark, manufacturer, or otherwise, does not necessarily constitute or imply its endorsement, recommendation, or favoring by the United States Government or the University of California. The views and opinions of authors expressed herein do not necessarily state or reflect those of the United States Government or the University of California, and shall not be used for advertising or product endorsement purposes. 


\title{
Monte Carlo simulation of the $R / 1$ automated damage test
}

\author{
Mike Runkel \\ Lawrence Livermore National Laboratory \\ P.O. Box 808, L-250 \\ Livermore, CA 94550 \\ Phone: (925)-424-2210 \\ FAX: (925)-422-3361 \\ E-mail: runkel1@1lnl.gov
}

\begin{abstract}
In this paper, a Monte Carlo computer analysis of the $\mathrm{R} / 1$ automated damage test procedure currently in use at LLNL is presented. This study was undertaken to quantify the intrinsic sampling errors of the R/1 ADT method for various types of optical materials, particularly KDP and fused silica, and to provide a recommended minimum number of test sites. A gaussian/normal distribution of $10 \mathrm{~J} / \mathrm{cm}^{2}$ average fluence $(\mu)$ was used as a damage distribution model. The standard deviation $(\sigma)$ of the distribution was varied to control its shape. Distributions were simulated which correspond to the damage distributions of $\operatorname{KDP}(\mu / \sigma=5-10)$ and fused silica $(\mu / \sigma \sim 15)$. A measure of the variability in test results was obtained by random sampling of these distributions and construction of the cumulative failure probability " $S$ " curves. The random samplings were performed in runs of 100 "tests" with the number of samples (i.e. sites) per test ranging from 2 to 500 . For distributions with $\mu / \sigma=5-10$, the study found an intrinsic error of 3 to $5 \%$ in the maximum deviation from the distribution average when using 100 site tests. The computations also showed substantial variation in the form of the CFD for any given test.

The simulation results were compared to actual data from eight 100 site $R / 1$ automated tests on a sample from rapidly grown KDP. It was found that while each 100 site damage probability curve could be fit to a gaussian distribution reasonably well, the 800 site cumulative damage probability curve was better modeled by a lognormal distribution. The differences observed in the individual CFD curves could be accounted for by sampling errors calculated from gaussian models.
\end{abstract}

Keywords: Monte Carlo simulation, damage testing, KDP, fused silica

\section{INTRODUCTION}

Within the last two years damage test procedures at LLNL have been extensively revised. The old standard S/1 (unconditioned) and R/1 (conditioned) damage tests have been abandoned because of the need to obtain larger, more statistically significant data sets which are used to help in understanding damage mechanisms, damage evolution and optic survivability on the National Ignition Facility (NIF) ${ }^{1,2,3}$. The R/1 automated damage test (ADT) which has evolved to replace the previous tests has the practical capacity to sample up to two orders of magnitude more area or volume in a particular optic. By testing each site to failure a cumulative failure distribution (CFD) can be constructed. By taking the derivative of the CFD, i.e. developing the histogram of the CFD appropriately, a damage probability curve can also be obtained. The damage probability curve provides insight into the number of damage mechanisms for a particular optic or material. For example, in the case of KDP we have found that the best samples still contain a low fluence tail resulting from defects which cannot be removed from the crystals by solution preparation, filtration and thermal or laser conditioning. It also allows failure probabilities to be calculated using various NIF model spatial profiles.

While the R/1 ADT method has proved to be a great step forward in our damage test capabilities, it is not without limitations. Intrinsic to any test procedure is an error which arises because the damage distribution cannot be completely sampled in a practical manner. There are many reasons (e.g. time, cost, risk, level of allowable destruction), depending on the specific circumstances of the optic under test, where an extensive damage test is impractical and may in fact be undesirable. In addition, each type of optic has specific test requirements and different damage morphologies. For example, bulk damage dominates in KDP with good quality diamond turned surfaces, and 
once formed, damage does not propagate in size until extremely high fluences are reached. This allows a large volume to be tested with many sites which are closely packed together. On the other hand, surface and (sub)surface damage dominates for fused silica and there are a number of effects related to the finishing process such as damage at scratches, the formation of gray haze and the onset of pinpoints. Once formed, however, surface damage on fused silica tends to propagate in size very rapidly with ejecta which can contaminate the surfaces for large distances around the test site. The effects are particularly bad at $355 \mathrm{~nm}(3 \omega)$ and require that the test site spacings be large. Consequently there is effectively much less available area for the typical battery of damage tests on a fused silica silica optic. In making the most effective use of the available test area or volume it is imperative to know how much testing is adequate to provide the information needed. One area of particular importance is the behavior of the damage distribution's low fluence tail in calculating failure probabilities via reliability analysis techniques ${ }^{3}$. This question is readily addressed in a computational fashion by Monte Carlo or random sampling methods ${ }^{4}$.

\section{MONTE CARLO DAMAge TESTING}

As an aid to understanding the results of the Monte Carlo analysis, it is helpful to consider the R/1 ADT method as a random sampler of the unknown, but well defined, damage distribution of the optic. Because none of the ADT systems at LLNL have stringent criteria for rejecting a site prior to test, the site grid chosen by the operator essentially represents a random sampling of local thresholds of the damage distribution. If there are no aberrations during the test, such as premature failures by contamination from a previous test site, the damage test data represent a subset of the actual distribution for the optic. However, problems arise when the data is interpreted. It is at best difficult to tell if features in the CFD represent the actual distribution or are artifacts of sampling. Two important features are kinks or shifts in the midrange portion of the curves and low fluence roll-off towards zero failure probability. The experimental solution to this dilemma is to repeat the test and compare the two curves. The degree of similarity of the curves quantifies test accuracy and allows construction of a more complete CFD. However, if experimental conditions do not allow further testing then "levels of confidence" must be assigned to the data and a certain level of uncertainty must be accepted.

The level of confidence in a given CFD curve can be established by using computational random sampling or Monte Carlo analysis. Over the course of extensive damage testing during NIF optics development, it has been noted that the basic damage probability curve (derived from the CFD) is well-enough ${ }^{5}$ represented by a gaussian probability distribution for a variety of optics. For this study it is convenient to characterize the shape of the distribution by the ratio of mean to standard deviation $(\mu / \sigma)$. In particular $R / 1$ automated test data has shown that $\mu / \sigma$ ratios range from 5 to 10 for KDP and are approximately 15 for fused silica, indicating that fused silica damage probability distributions are more sharply peaked. The normalized gaussian distribution has the form

$$
P(F)=\frac{1}{\sigma \sqrt{2 \pi}} \exp \left[-(\mu-F)^{2} / 2 \sigma^{2}\right]
$$

where $\mu$ and $\sigma$ denote the mean and standard deviation respectively.

In contrast to the R/1 ADT where the cumulative failure distribution is sampled and the failure probability curve derived from it, Monte Carlo analysis assumes a given failure probability curve and builds the CFD from random samplings of it. In order to mimic the R/1 ADT method, variations in the CFD curves were quantified by exploring a matrix where the variable parameters were $\mu / \sigma$ and $N_{\text {sites }}$, the number of samples per test. This is analogous to testing different optics with different number of test sites. In particular, the values of $\mu / \sigma$ were $2,5,10,15$ and 20 giving failure probability curves ranging from very broad to very peaked. $\mathrm{N}_{\text {sites }}$ values were $2,5,10,15,25,50$, 100,250 and 500 . The resulting CFD "S" curves were analyzed for min, max, mean and standard deviation. To ensure an adequate sampling of the possible CFD curves, the "test" was repeated 100 times for each $\mu / \sigma, N_{\text {sites }}$ pair. It was then possible to statistically analyze the data from each CFD curve and compare it to the generating distribution. Figure 1 illustrates the sampling method. 

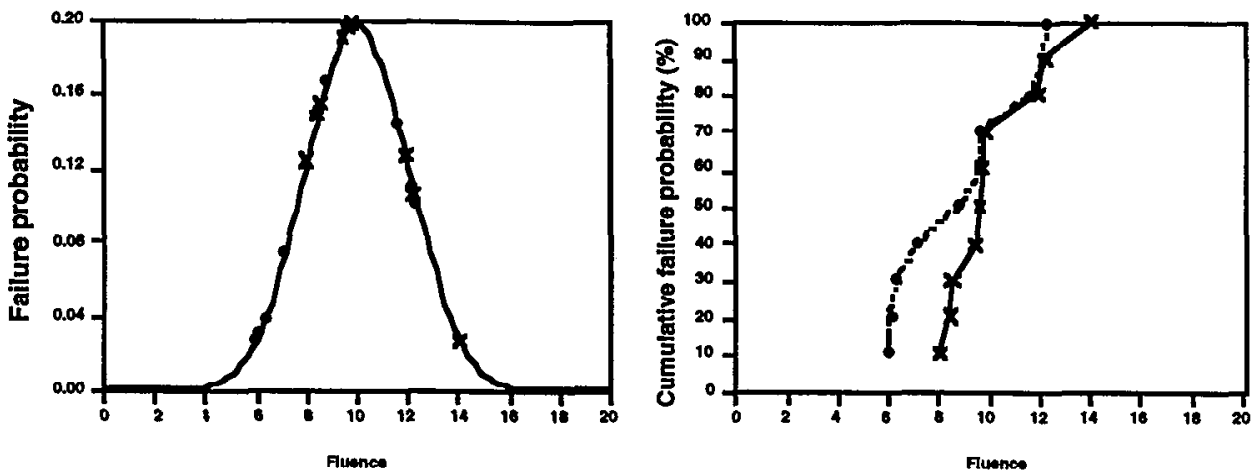

Figure 1. Two 10 site random samplings of a gaussian distribution of $10 \pm 2 \mathrm{~J} / \mathrm{cm}^{2}$ and their corresponding cumulative failure distributions.

For the sake of easy conceptualization, the mean of the distribution was chosen to be $10 \mathrm{~J} / \mathrm{cm}^{2}$. The cases of $\mu / \sigma=$ 5,10 and 15 are of particular interest because they correspond to typical distributions seen in KDP $(\mu / \sigma=5-10)$ and fused silica $(\mu / \sigma \sim 15)$, as mentioned above.

\subsection{Simulation results:}

To obtain a picture of the behavior of the test data as a function of $\mathrm{N}_{\text {sites }}$, the mean and standard deviation of each CFD was plotted for the 100 tests performed/computed. Figure 2 shows results for variations in the mean and standard deviation of each individual CFD for the case where $\mu / \sigma=5$. This corresponds approximately to the distribution derived from rapidly grown RG7B KDP crystals. There is significant fluctuation in the 5 sites/test regime with the CFD averages deviating by up to $\pm 22 \%$ from the actual distribution average and up to $14 \%$ for the CFD standard deviations. It should be noted that the old 10 site $\mathrm{R} / 1$ test method (i.e. threshold determined by 5 undamaged and 5 damaged sites) falls into this regime. As $\mathrm{N}_{\text {sites }}$ increases the mean and standard deviations converge toward the actual values of the distribution. When $N_{\text {sites }}=25$ they are roughly half those for $\mathbf{N}_{\text {sites }}=5$. The error drops by half again when $\mathrm{N}_{\text {sites }}=100$. By the time $\mathrm{N}_{\text {sites }}=500$ the maximum average error is on the order of $2 \%$.
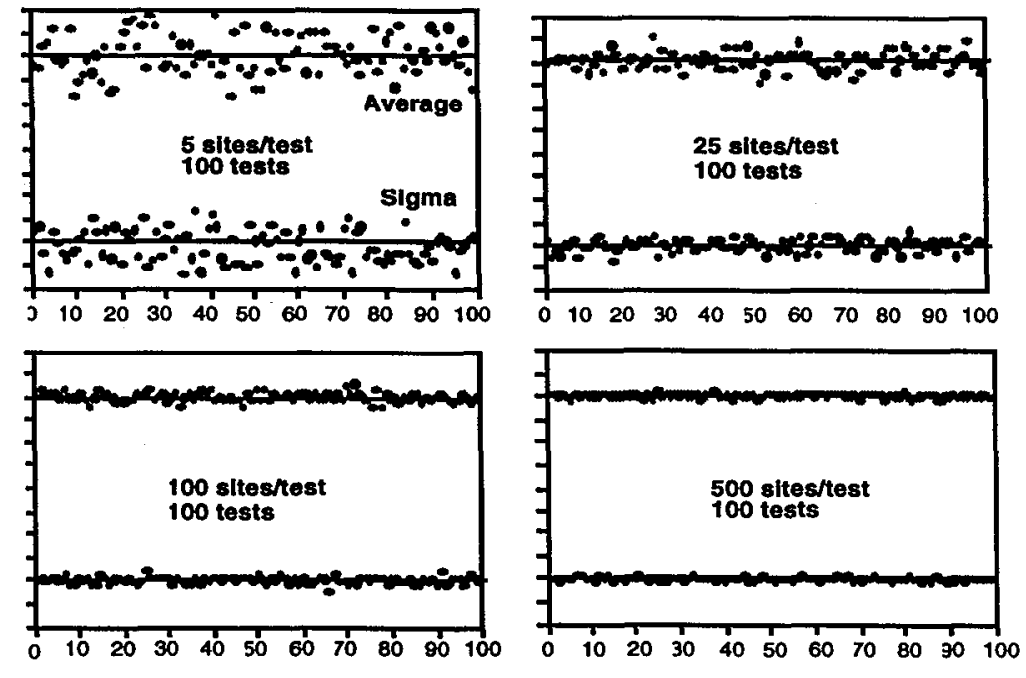

rest number

Figure 2. Mean and standard deviation data taken from cumulative failure probability curves for the case of $\mu=10$ $\mathrm{J} / \mathrm{cm}^{2}$ and $\sigma=2$. Deviations in the mean and standard deviation of each CFD are on the order of 5 percent for $\mathrm{N}_{\text {sites }}=$ 100 . 
The convergence of this data can also be plotted as shown in Figure 3. Here the mean and standard deviation of each CFD are plotted for a given value of $\mathrm{N}_{\text {sites }}$. The logarithmic scale readily shows the convergence of the CFDs to the distribution values as $\mathbf{N}_{\text {sites }}$ increases.

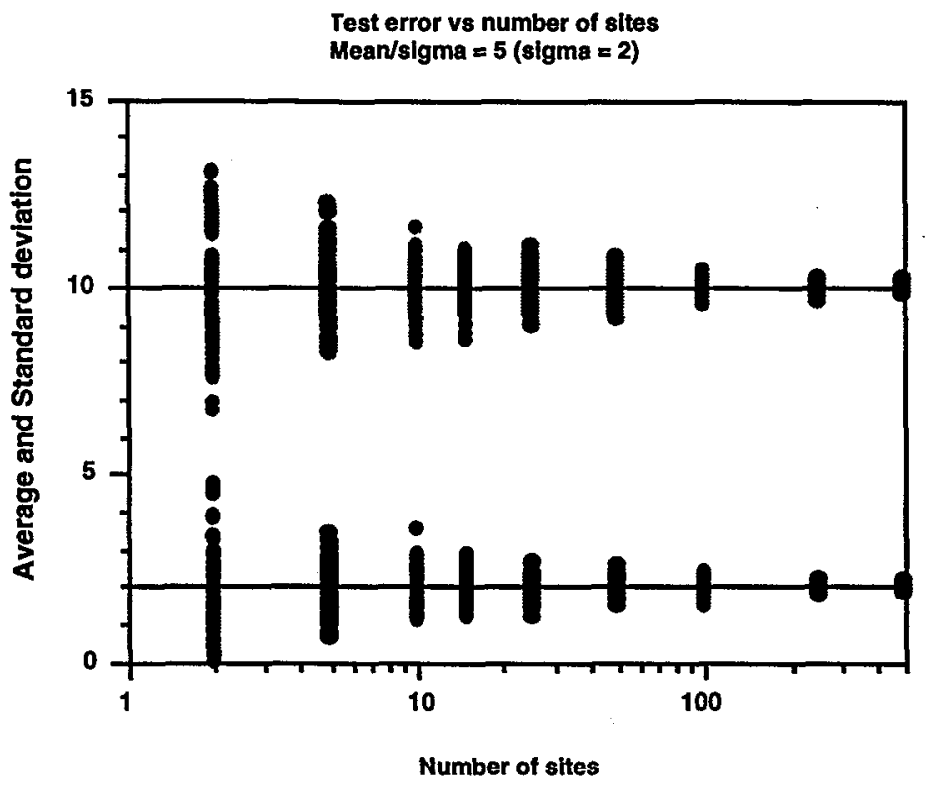

Figure 3. Plot of the variation in the mean and standard deviation versus $N_{\text {sites }}$ for a $\mu / \sigma=5$ distribution. This is the same data as shown in Figure 2.

Numerical values for the data are given in Table 1. The tabulated values include the maximum deviation of the individual CFD averages from the distribution average, the average deviation of all the CFD curves from the distribution average, the maximum deviation of the individual CFD standard deviations from the distribution average and the average deviation of the individual CFD standard deviations from the distribution standard deviation. Bar plots and tabulated data for all values of $\mu / \sigma$ and $\mathrm{N}_{\text {sites }}$ simulated are given in Appendix 1 .

Table 1. Statistical parameters for the case of $\mu / \sigma=5$. Max $\left|\mu-F_{i}\right|$ refers to the largest deviation of an individual CFD curve average from the actual distribution mean. Avg $\left|\mu-F_{i}\right|$ denotes the average deviation of the entire data set from the distribution mean. Max| $\sigma-\sigma_{i} \mid$ and Avg| $\sigma-\sigma_{i} \mid$ are analogous quantities for the standard deviation.

Percent deviations from the distribution average and standard deviation for $\mu / \sigma=5$.

\begin{tabular}{|c|c|c|c|c|}
\hline $\mathbf{N}_{\text {sites }}$ & Max $\left|\mu-F_{i}\right|$ & Avg $\left|\mu-F_{i}\right|$ & $\operatorname{Max}\left|\sigma-\sigma_{i}\right|$ & Avglo- $\sigma_{i} \mid$ \\
\hline 2 & 32.6 & 11.4 & 27.5 & 10.5 \\
\hline 5 & 21.9 & 7.2 & 14.0 & 6.0 \\
\hline 10 & 15.7 & 4.9 & 15.2 & 3.4 \\
\hline 15 & 13.9 & 3.7 & 8.8 & 2.8 \\
\hline 25 & 11.0 & 3.0 & 8.0 & 2.3 \\
\hline 50 & 8.2 & 2.5 & 5.4 & 1.7 \\
\hline 100 & 5.0 & 1.5 & 4.7 & 1.1 \\
\hline 250 & 3.5 & 0.9 & 2.1 & 0.7 \\
\hline 500 & 1.9 & 0.7 & 1.6 & 0.5 \\
\hline
\end{tabular}

To obtain an idea of the degree of differences which are possible between the CFD curves, a number of them ane plotted in Figure 4. It shows the four curves of the $\mu / \sigma=5, N_{\text {sites }}=100$ data which exhibit the highest and lowest mean and standard deviations relative to the actual distribution. 


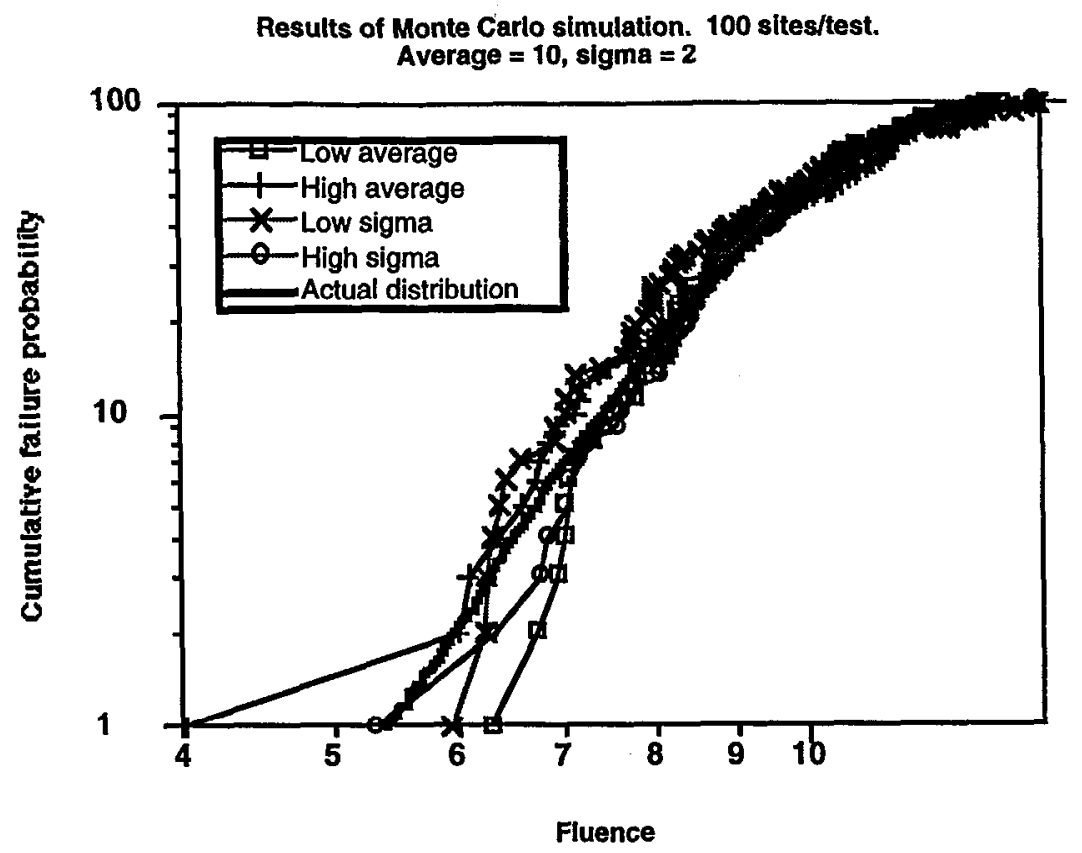

Figure 4. Log-log plot of cumulative failure distributions for tests exhibiting the lowest and highest mean and standard deviation relative to the actual distribution. The logarithmic axes enhance the differences between the curves, especially at lower fluence/failure probability levels.

The plot shows that the CFDs do not converge well to the actual distribution until failure probabilities of $50 \%$ ane reached. Thus for the case of material similar to rapidly grown KDP RG7B-52 we see that even 100 site testing may yield widely varying results. Increasing the number of sites to 250 yields reasonable convergence at CFD values of 2\% (Figure 5).

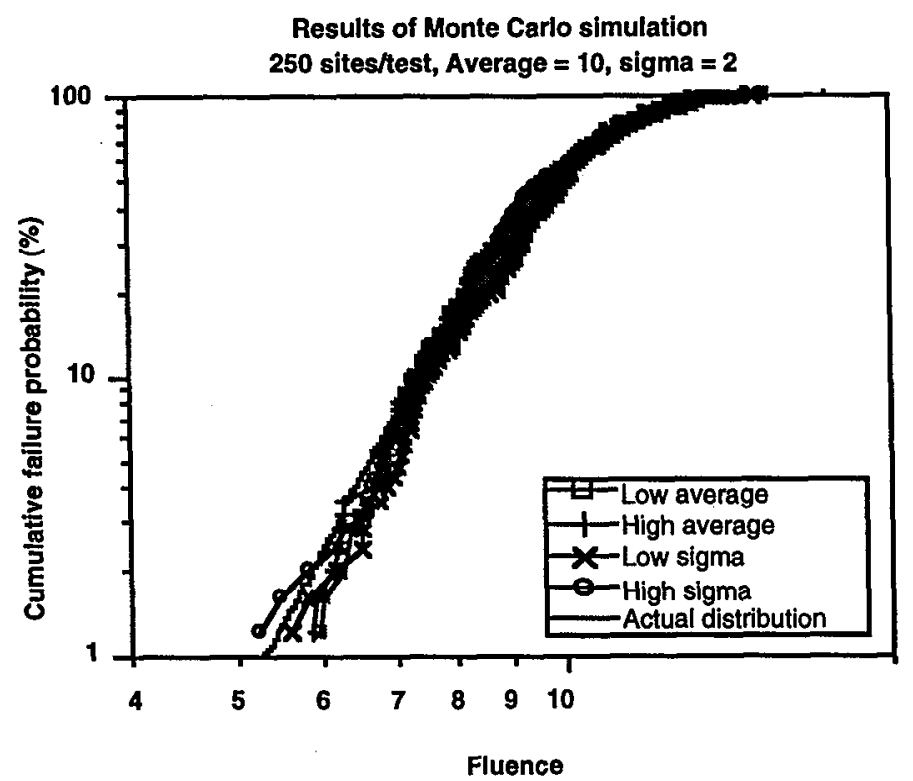

Figure 5. Log-log CFD plot for the $\mathrm{m} / \mathrm{s}=5$ and $\mathrm{N}_{\text {sites }}=250$. Convergence to the actual distribution occurs when CFD values are greater than $2 \%$.

The other practical cases of interest are the normal 16 and 100 site tests applied to fused silica. Figure 6 shows a $\log \log$ plot of the CFD versus fluence for $\mu / \sigma=15$ and $N_{\text {sites }}=15$ while Figure 7 shows a similar plot for the $-N_{\text {sites }}$ $=100$ case. 


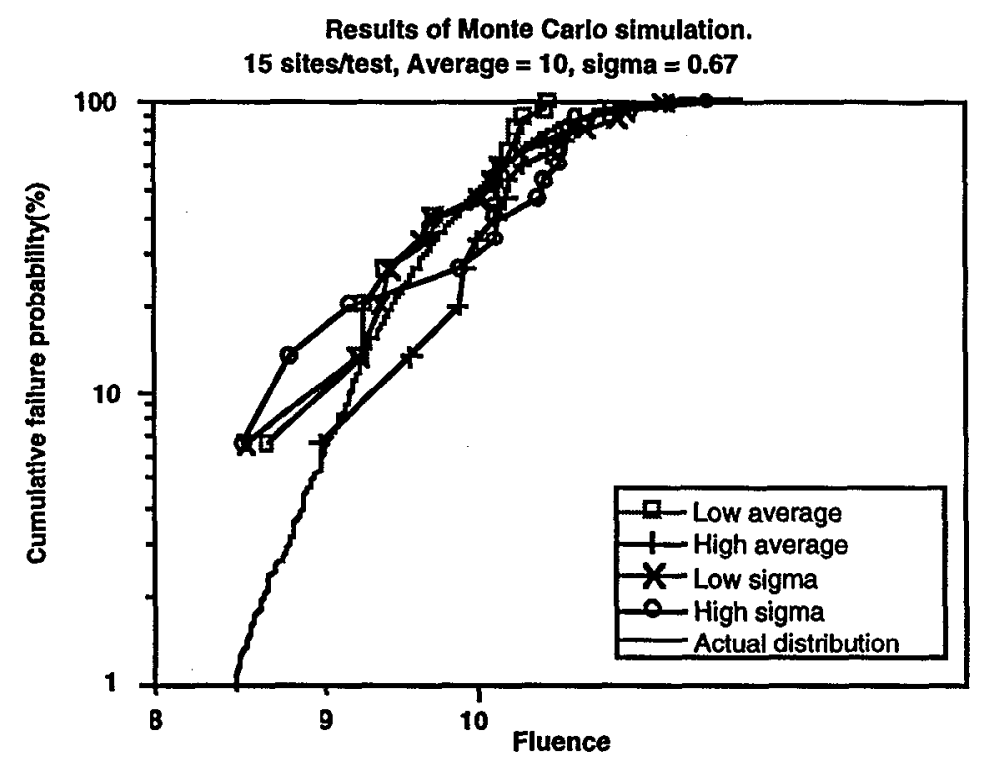

Figure 6. $\log -\log$ plot of CFD vs. fluence for 15 site test representative of fused silica $(\mu / \sigma=15)$.

The curves do not converge to the actual distribution until CFD values exceed $80 \%$. In the case of the low average curve there is very little similarity to the generating curve.

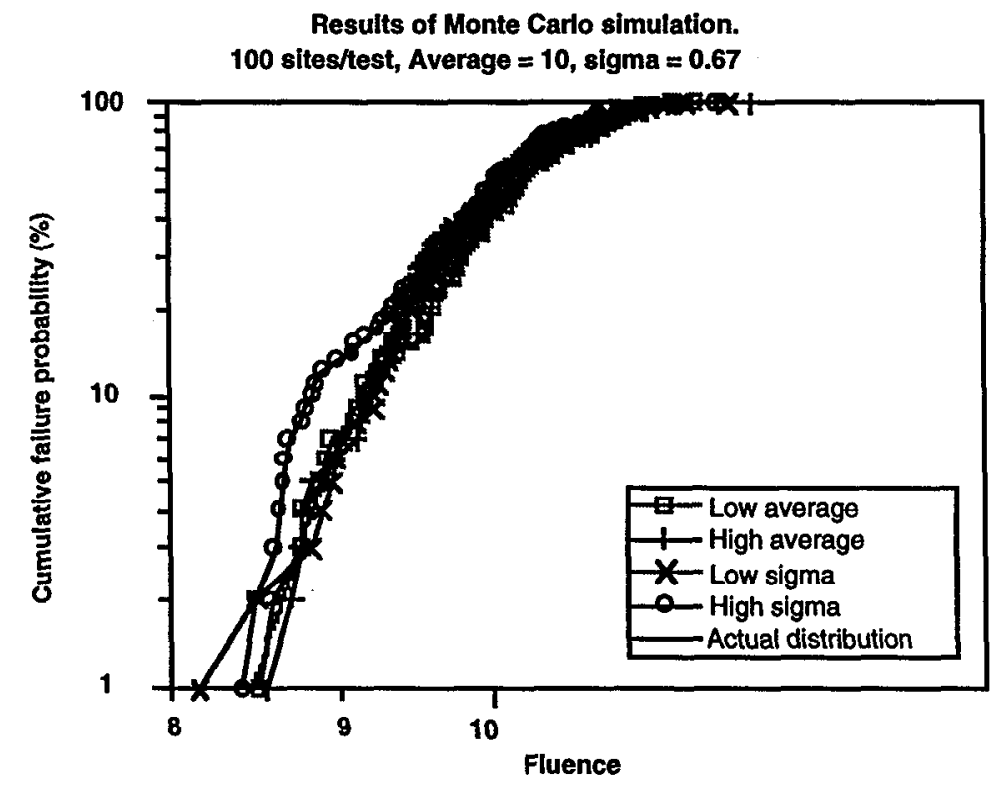

Figure 7. $L$ og-log plot of CFD vs. fluence for 100 site tests on fused silica $(\mu / \sigma=15)$. The curves converge toward the actual distribution when CFD values exceed 30\%. With the exception of the high sigma curve convergence takes place at CFD values above $3 \%$.

\subsection{Determination of the number of sites based on desired levels of confidence:}

In this section two prescriptions are provided for determining the number of sites needed in a particular test to achieve a desired level of confidence. The first is concerned with determining whether shifts in the mean value of a $\mathrm{CFD}$ are real. This would correspond to situations such as thermal treatment of $\mathrm{KDP}$ or acid etching of fused silica. 
Initially an R/1 ADT of $\mathrm{N}_{\text {sites }}$ must be performed. The CFD and $\mu / \sigma$ must be determined. After this, the tables of Appendix 1 can be used to determine the level of uncertainty expected in the data. If the expected level of accuracy for the test is greater than the desired error, the additional number of sites is determined by looking up (or interpolating) the number of test sites giving the desired level of accuracy. The optic must then be tested until the required number of sites has been reached. The analysis is then repeated to determine if the required level of accuracy has been reached.

The second prescription deals with the low fluence roll-off region of the CFD curve. As we have seen, it is possible to have significant variation in the lower fluence portion of a CFD despite relatively high $\mathbf{N}_{\text {sites }}$ values and therefore the level of confidence in this region is low. The test operator is faced with two problems. The first is to determine the possible error in the low fluence portion of the curve for the sites already tested, and whether this error is acceptable. The second, is to determine the number of additional test sites required to bring the sampling error of the CFDs lower portion into the acceptance criterion. To address the first problem it is necessary to determine the mean and standard deviation of the existing CFD. Next, the number of sites in the low fluence portion, i.e. outside the standard deviation, of the curve are counted. Tables in Appendix 1 can be interpolated to determine an expected error value for the number of low fluence sites and the calculated $\mu / \sigma$ value. If the error exceeds the acceptance criterion, the number of additional test sites can also be determined from the tables. Interpolation of the tables yields the number of additional test sites which must be added to the low fluence portion of the curve to attain the desired level of confidence. But, because of the gaussian nature of the damage probability distribution only one sixth of the data, on average, will fall on the lower portion of the curve outside of the standard deviation. Therefore, 6 times as many sites must be added as were originally calculated.

\section{RESULTS OF 8X100 DAMAGE TESTING ON RAPIDLY GROWN KDP RG7B:}

Because of the intrinsic sampling error and difficulty in interpreting a single 100 site CFD for KDP like simulations, an extensive battery of tests on sample RG7B-52 were performed by repeating the 100 site R/1 ADT until a significant portion of the test sample had been probed. In all, eight 100 site tests were performed. This allowed investigation of a number of issues which had been unexplored. They included 1) comparing real data to the Monte Carlo simulation, 2) looking for variations in damage threshold across sector boundaries (in this case there was a prism/prism or pyramid/pyramid boundary in the sample) and 3) determining whether the damage statistics were well modeled by a gaussian distribution. The $5 \times 5 \times 1 \mathrm{~cm}$ sample was obtained from one of the first rapidly grown, $50 \mathrm{~cm}$ class boules and is relevant because it represents the type of material to be used for NIF Pockel's cells and second harmonic generation crystals.

Figure 8 shows the eight individual and cumulative CFD curves in standard and log-log formats. It can be seen immediately that the damage behavior is different than for the simulation. The deviation of the curves from the cumulative value is substantially larger for the real data than for the computed cases. Whereas the simulated curves tend to converge toward the cumulative distribution at high CFD values, the actual data shows a high degree of divergence. Table 1 gives the statistics for min, max, mean and standard deviation for each of the curves and shows a large variation in the $\mu / \sigma$ ratios for each individual test. Ultimately the cumulative $\mu / \sigma$ value was 3.4 , which is significantly different from the $\mu / \sigma$ ratio of 5 assumed at the onset of modeling. As a result, this case was also modeled to provide a better comparison to the real data. The modeling data is shown in Figure 9. 

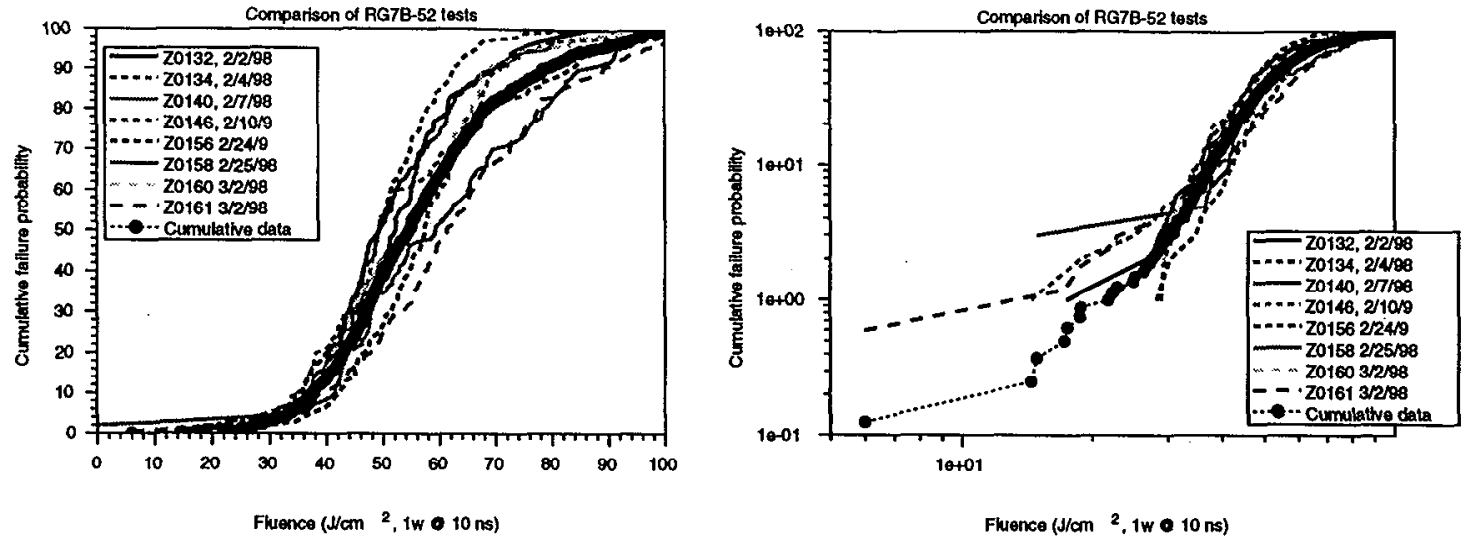

Figure 8. Cumulative failure distribution plots for sample RG7B-52 in normal and log-log format.

Table 1. Test data for the individual curves of RG7B-52. Fluence values are for $1064 \mathrm{~nm}$ pulses with $10 \mathrm{~ns}$ pulsewidths.

\begin{tabular}{|c|c|c|c|c|c|}
\hline Test number & $\begin{array}{c}\text { Minimum } \\
\text { fluence } \\
\left(\mathrm{J} / \mathrm{cm}^{2}\right)\end{array}$ & $\begin{array}{c}\text { Maximum } \\
\text { fluence }\left(\mathrm{J} / \mathrm{cm}^{2}\right)\end{array}$ & $\begin{array}{c}\text { Mean } \\
\text { fluence }\left(\mathrm{J} / \mathrm{cm}^{2}\right)\end{array}$ & $\begin{array}{c}\text { Standard deviation } \\
\left(\mathrm{J} / \mathrm{cm}^{2}\right)\end{array}$ & $\begin{array}{c}\text { Mean/standard } \\
\text { deviation }\end{array}$ \\
\hline \hline $\mathrm{Z} 0132$ & 29.0 & 82.8 & 49.5 & 10.7 & 4.6 \\
\hline $\mathrm{Z} 0134$ & 17.5 & 83.6 & 51.4 & 12.8 & 4.0 \\
\hline $\mathrm{Z} 0140$ & 22.3 & 86.6 & 52.6 & 13.1 & 4.0 \\
\hline $\mathrm{Z} 0146$ & 14.5 & 91.1 & 52.6 & 15.2 & 3.5 \\
\hline Z0156 & 28.3 & 108.0 & 59.6 & 15.4 & 3.9 \\
\hline Z0158 & 25.2 & 96.3 & 60.4 & 19.7 & 3.1 \\
\hline Z0160 & 19.8 & 145 & 61.5 & 21.6 & 2.8 \\
\hline Z0161 & 6.0 & 133 & 63.2 & 20.1 & 3.1 \\
\hline Cumulative & 6.0 & 145 & 56.4 & 16.5 & 3.4 \\
\hline
\end{tabular}

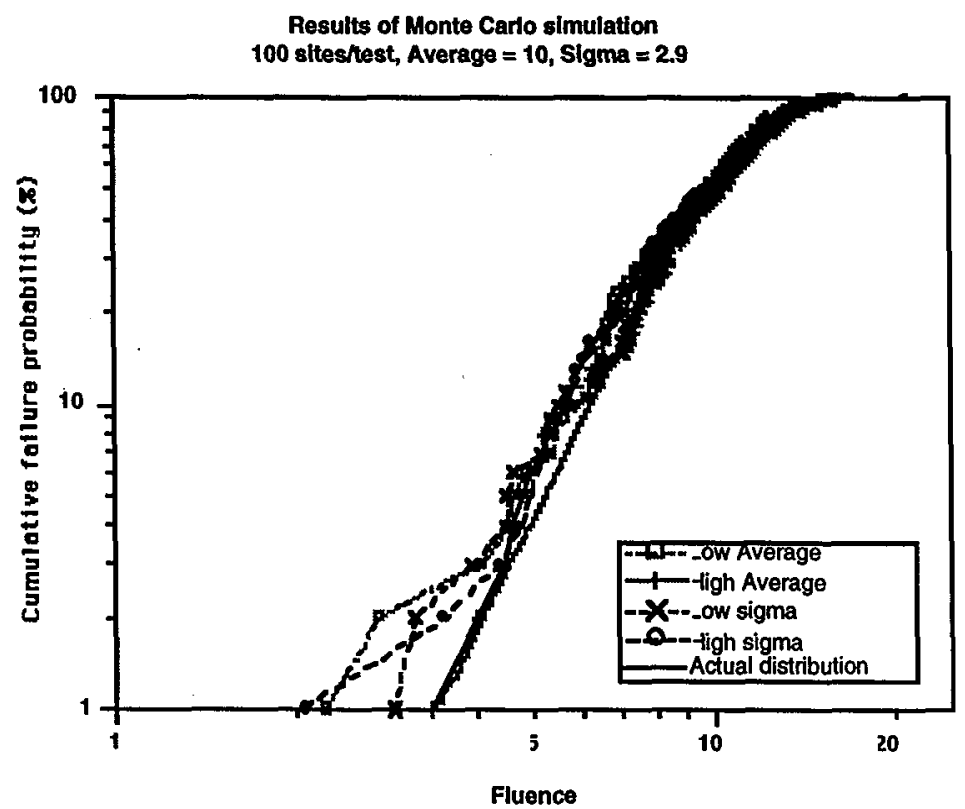

Figure 9. Results of Monte Carlo simulation for $\mu / \sigma=3.4$. This value was chosen to correspond to the average value obtained for the 800 site test on RG7B-52. 
The maximum deviation from the cumulative mean is $\pm 12 \%$, seen in tests Z0132 and Z0161. In contrast the Monte Carlo model for $\mu / \sigma=3.4$ predicts a maximum deviation from the distribution mean of $8 \%$. It also predicts that the curves will converge to the mean at CFD values of 50\%. For the actual test data the curves converge at CFD values of approximately 5\%, although there is still significant variation in the test data beyond this value. The fact that the gaussian simulation does not account for the observed variation in the individual test curves becomes clear when the damage probability distribution is derived for the total 800 sites. As shown in Figure 10 this curve fits a lognormal distribution ${ }^{5,6}$ quite well.

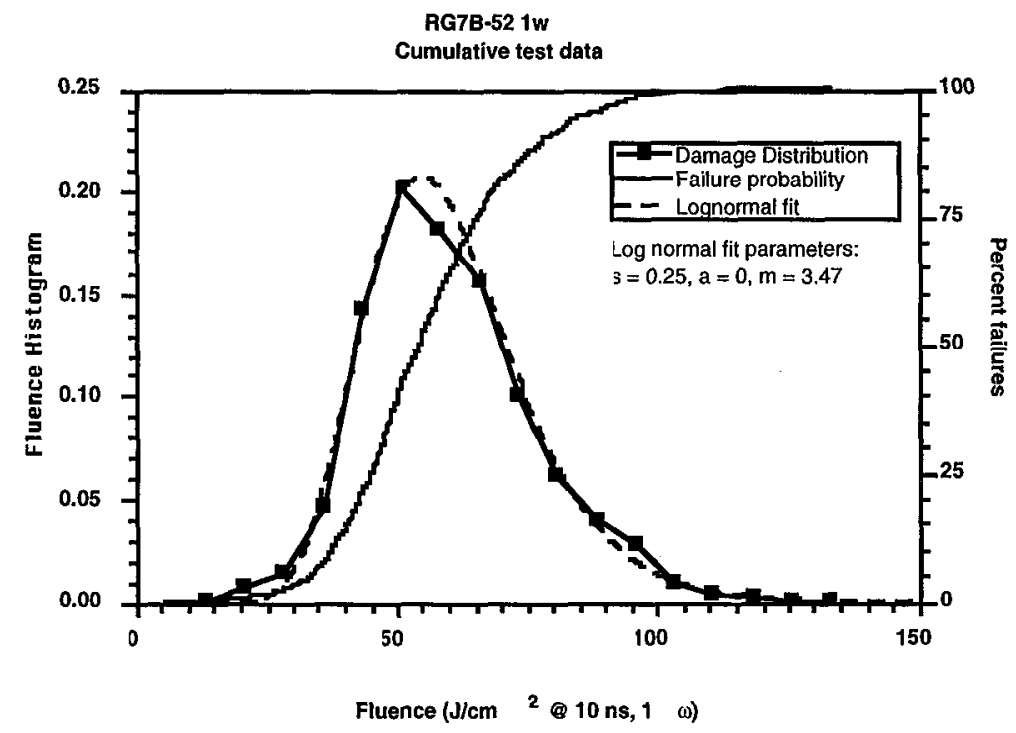

Figure 10. Cumulative failure distribution and corresponding damage probability curve for the 800 sites tested on RG7B-52. The damage probability curve is well modeled by a lognormal distribution.

With this knowledge the behavior of the individual curves can be easily explained. As was seen in the simulations, the more peaked distributions required less sampling to achieve the same level of confidence as broader distributions. For the RG7B data, the steeply rising portion of the curve is better sampled than the broader, slowly decaying tail. This difference in sampling resolution accounts for the rapid convergence of the individual CFD curves at the 5\% CFD (log-log plot) value as well as the large differences in the shapes of the curves for CFD values from 30-99\%. While the lognormal distributions have not been modeled via Monte Carlo methods, the error expected in each part of the curve can be approximated by fitting the leading edge of the curve with a narrow gaussian and the trailing edge with a broader gaussian. For the leading edge a gaussian of $\mu / \sigma=52 / 10=5.2$ fits well. From this we expect sampling errors of $8 \%$ for the fifty or so sites in the leading edge. For the trailing edge a gaussian of $\mu / \sigma=52 / 19=2.7$ fits well. This gives a fifty site maximum sampling error of $17 \%$ based on linear interpolation of the tables.

Finally, the 800 site test allowed determination of whether sector boundaries affected the damage behavior. Sample RG7B-52 contained a pyramid/pyramid or prism/prism sector boundary which was readily observed in interferometry or polarimetry. Figure 11 shows an intensity plot representing the local damage threshold for the grid tested. There are no indications of abnormal damage behavior in the vicinity of the sector boundary. While this test shows that there are no apparent differences in damage behavior across sector boundaries of the same type, the question of damage behavior across a prism/pyramid boundary remains open. This question is much more interesting because each sector incorporates cationic impurities, particularly $\mathrm{Fe}^{3+}$, at much different levels. Future plans call for investigation of this question by testing samples with equal areas of prism and pyramid sectors. 


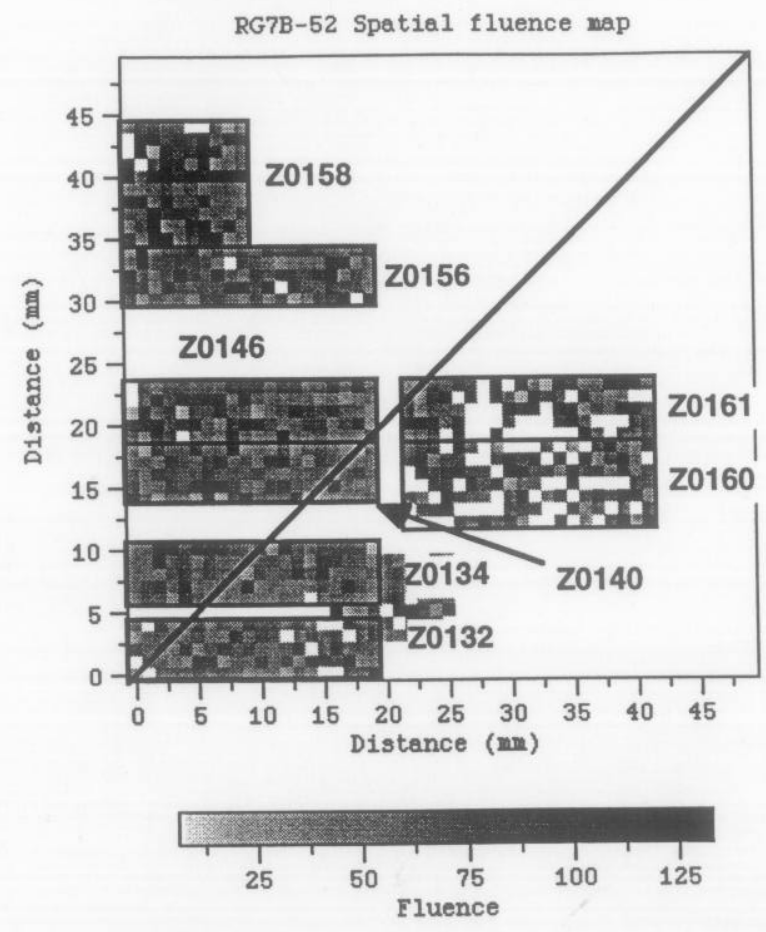

Figure 11. Spatial fluence map of RG7B-52 indicating the areas tested and where the sector boundary runs. Data taken outside of marked test areas was not included in the analysis. There is no apparent indication of unusual damage behavior near the boundary. The blank white squares within a test box indicate sites which were rejected because of surface contamination.

For $\mu / \sigma=5-10,100$ site tests undersample the actual damage distribution and the lower fluence portion of the curve has substantial error due to the statistically low number of sites. 250 site test increase the accuracy of the test substantially so that the CFD curves converge toward the actual distribution at failure probabilities around $2 \%$ instead of $50 \%$.

For $\mu / \sigma=15$ and 15 site tests, the individual CFD curves show dramatic variation in shape converging to the actual distribution at CFD values in excess of $80 \%$. Consequently, they yield virtually no reliable information regarding the actual distribution. Increasing the number of sites to 100 yields substantially better accuracy. In this case, convergence of the CFD values occurs at $20 \%$.

Tabulated data from the simulations can be used to determine the intrinsic error in actual test data. By following procedures outlined above, the test operator can determine the additional number of test sites required to obtain any desired level of sampling accuracy.

Simulation data can be used to predict expected behavior for lognormal distributions by fitting the leading and trailing edges with different shaped curves.

\section{GENERAL CONCLUSIONS}

The results of the Monte Carlo simulation together with the 800 site test revealed that there can be significant error present in any R/1 ADT of the type now conducted. Careful analysis of the test data is required to achieve the maximum usefulness from the test method. The basic recommendation for any test scenario is to test as many sites 
as possible. In the case of KDP it appears that 100 site tests undersample the typical distribution. Future tests will be conducted with a significantly greater number of test sites.

\section{$4.18 \times 100$ site test on RG7B-52 conclusions}

Each individual curve in the test showed substantial variation. A gaussian distribution could be made to fit any of the individual 100 site curves quite well. The lognormal character of the CFD was not revealed until roughly 500 sites had been probed. Consequently, the gaussian simulation for $\mu / \sigma=3.4$ underestimated the sampling error predicting errors of $8 \%$ while the actual errors were $12 \%$. As mentioned above the leading and trailing edges of the lognormal distribution were fit with different shaped gaussians. It was found that for the leading edge an error of $6 \%$ could be expected while the trailing edge maximum error was $17 \%$.

The spatial fluence map of the 800 test sites revealed no change in the damage behavior across like sector boundaries. The question of damage distribution changes across prism/pyramid boundaries will be addressed in future tests.

*Work performed under the auspices of the U.S. Department of Energy by Lawrence Livermore National Laboratory under Contract No. W-7405-ENG-48.

\section{REFERENCES}

1) M. Runkel, M. Yan, J. DeYoreo, N. Zaitseva "The effect of impurities and stress on the damage distributions of rapidly grown KDP crystals," in Laser Induced Damage in Optical Materials, SPIE Proceedings, Volume 3244, edited by G. Exarhos et. al., 211-222, SPIE, Bellingham, WA. 1997

2), M. Runkel, J. DeYoreo, W. Sell, D. Milam, "Laser conditioning study of KDP on the Optical Sciences Laser using large area beams," in Laser Induced Damage in Optical Materials, SPIE Proceedings, Volume 3244, edited by G. Exarhos et. al., 51-63, SPIE, Bellingham, WA. 1997

3) M. Runkel, W. Williams, J. DeYoreo, "Predicting NIF tripler damage from Zeus and OSL test resuits and NIF spatial profiles," to appear in Laser Induced Damage in Optical Materials, SPIE Proceedings, 1998

4) Numerical Recipes, W. Press et. al., Cambridge University Press, 1986

5) The functional form for the log normal distribution is

$$
P(F)=\frac{1}{\sigma(F-a) \sqrt{2 \pi}} e^{-\frac{[\ln (F-a)-m]^{2}}{2 \sigma^{2}}}
$$

where $\mathrm{F}$ represents the fluence, $\mathrm{a}, \sigma$ and $\mathrm{m}$ are fitting parameters. For the RG7B-52 data the numerical values are $\mathrm{a}=$ $0, \sigma=0.25, \mathrm{~m}=3.47$.

6) Mathematical Methods of Statistics, H. Cramer, Princeton University Press, 1946 


\section{APPENDIX 1}

Tabulated data for damage test simulations.

\begin{tabular}{|c|c|c|c|c|}
\hline \multicolumn{5}{|c|}{ Percent deviations from the distribution average and standard deviation for $\mu / \sigma=2}$. \\
\hline $\mathrm{N}_{\text {sites }}$ & Max $\left|\mu-\mathrm{F}_{i}\right|$ & Avg $\left|\mu-F_{i}\right|$ & Max $\left|\sigma-\sigma_{j}\right|$ & Avg $\left|\sigma-\sigma_{i}\right|$ \\
\hline \hline 2 & 77.9 & 26.1 & 119.6 & 27.2 \\
\hline 5 & 66.7 & 17.6 & 34.1 & 13.3 \\
\hline 10 & 38.2 & 13.1 & 25.6 & 9.4 \\
\hline 15 & 33.9 & 11.5 & 31.1 & 8.0 \\
\hline 25 & 30.2 & 7.8 & 15.1 & 5.5 \\
\hline 50 & 19.9 & 5.9 & 18.5 & 4.3 \\
\hline 100 & 10.5 & 4.1 & 8.2 & 2.9 \\
\hline 250 & 7.8 & 3.0 & 7.6 & 1.9 \\
\hline 500 & 4.9 & 1.9 & 3.3 & 1.3 \\
\hline
\end{tabular}

Percent deviations from the distribution average and standard deviation for $\mu / \sigma=5$.

\begin{tabular}{|c|c|c|c|c|}
\hline $\mathbf{N}_{\text {sites }}$ & $\operatorname{Max}\left|\mu-F_{j}\right|$ & Avg $\left|\mu-F_{i}\right|$ & $\operatorname{Max}\left|\sigma-\sigma_{i}\right|$ & Avg| $\sigma-\sigma_{i} \mid$ \\
\hline 2 & 32.6 & 11.4 & 27.5 & 10.5 \\
\hline 5 & 21.9 & 7.2 & 14.0 & 6.0 \\
\hline 10 & 15.7 & 4.9 & 15.2 & 3.4 \\
\hline 15 & 13.9 & 3.7 & 8.8 & 2.8 \\
\hline 25 & 11.0 & 3.0 & 8.0 & 2.3 \\
\hline 50 & 8.2 & 2.5 & 5.4 & 1.7 \\
\hline 100 & 5.0 & 1.5 & 4.7 & 1.1 \\
\hline 250 & 3.5 & 0.9 & 2.1 & 0.7 \\
\hline 500 & 1.9 & 0.7 & 1.6 & 0.5 \\
\hline
\end{tabular}

Percent deviations from the distribution average and standard deviation for $\mu / \sigma=10$.

\begin{tabular}{|c|c|c|c|c|}
\hline $\mathrm{N}_{\text {sites }}$ & $\operatorname{Max}\left|\mu-F_{i}\right|$ & Avg $\left|\mu-F_{i}\right|$ & $\operatorname{Max}\left|\sigma-\sigma_{j}\right|$ & Avglo- $\sigma_{j} \mid$ \\
\hline 2 & 18.6 & 6.1 & 18.6 & 5.4 \\
\hline 5 & 13.8 & 3.7 & 8.0 & 2.7 \\
\hline 10 & 8.7 & 2.4 & 5.3 & 2.0 \\
\hline 15 & 6.0 & 2.1 & 4.5 & 1.5 \\
\hline 25 & 6.1 & 1.7 & 4.2 & 1.1 \\
\hline 50 & 4.3 & 1.3 & 3.1 & 0.9 \\
\hline 100 & 2.9 & 0.7 & 1.9 & 0.5 \\
\hline 250 & 1.4 & 0.5 & 1.1 & 0.4 \\
\hline 500 & 1.6 & 0.4 & 0.7 & 0.3 \\
\hline
\end{tabular}


Percent deviations from the distribution average and standard deviation for $\mu / \sigma=15$.

\begin{tabular}{|c|c|c|c|c|}
\hline$N_{\text {sites }}$ & Maxl$\left|\mu-F_{i}\right|$ & Avg $\left|\mu-F_{i}\right|$ & $\operatorname{Max}\left|\sigma-\sigma_{j}\right|$ & Avg $\left|\sigma-\sigma_{j}\right|$ \\
\hline 2 & 13.6 & 3.2 & 13.5 & 3.1 \\
\hline 5 & 7.6 & 2.4 & 5.4 & 2.0 \\
\hline 10 & 6.0 & 2.0 & 4.0 & 1.2 \\
\hline 15 & 6.4 & 1.4 & 3.3 & 0.8 \\
\hline 25 & 4.4 & 1.1 & 2.4 & 0.7 \\
\hline 50 & 2.2 & 0.7 & 1.6 & 0.4 \\
\hline 100 & 1.8 & 0.6 & 1.1 & 0.4 \\
\hline 250 & 1.1 & 0.4 & 1.1 & 0.2 \\
\hline 500 & 0.8 & 0.2 & 0.6 & 0.2 \\
\hline
\end{tabular}

Percent deviations from the distribution average and standard deviation for $\mu / \sigma=20$.

\begin{tabular}{|c|c|c|c|c|}
\hline $\mathbf{N}_{\text {sites }}$ & $\operatorname{Max}\left|\mu-F_{i}\right|$ & Avg $\left|\mu-F_{i}\right|$ & $\operatorname{Max}\left|\sigma-\sigma_{i}\right|$ & 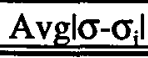 \\
\hline 2 & 10.6 & 3.06 & 8.52 & 2.73 \\
\hline 5 & 5.64 & 1.68 & 3.66 & 1.37 \\
\hline 10 & 4.07 & 1.29 & 2.90 & 0.93 \\
\hline 15 & 2.95 & 0.92 & 2.75 & 0.74 \\
\hline 25 & 2.72 & 0.81 & 1.91 & 0.59 \\
\hline 50 & 1.85 & 0.64 & 1.12 & 0.41 \\
\hline 100 & 1.60 & 0.40 & 0.91 & 0.31 \\
\hline 250 & 0.88 & 0.27 & 0.60 & 0.18 \\
\hline 500 & 0.53 & 0.18 & 0.62 & 0.13 \\
\hline
\end{tabular}

